\title{
Initial Background Assessment for Cosmic Dust Collection at Mauna Loa Observatory
}

\author{
A. C. GENABE ${ }^{1,2}$, H. A. ISHII ${ }^{1}$, J.P. BRADLEY ${ }^{1}$, \\ L. S. ALESBROOK ${ }^{3}$, P. J. WOZNIAKIEWICZ ${ }^{3}$ \\ ${ }^{1}$ Hawaii Institute of Geophysics \& Planetology, University of \\ Hawai'i at Mānoa, USA \\ ${ }^{2}$ Dept. of Earth Sciences, University of Hawai'i at Mānoa \\ ${ }^{3}$ School of Physical Sciences, University of Kent, UK
}

Dust-producing bodies in our solar system like comets and asteroids release particles of extraterrestrial (ET) material commonly known as cosmic dust. Approximately 40,000 tons of these particulates reach Earth's surface annually and a variety methods have been used in prior cosmic dust collection efforts. However, current methods of cosmic dust collection face multiple challenges, which include the obscuring effects of terrestrial dust, leaching effects caused by prolonged exposure to snow, ice, and water, and various forms of contamination during collection.

We are collecting cosmic dust at the Mauna Loa Observatory (MLO) on the island of Hawai'i using a highvolume air sampler to capture particles directly from air onto ultra clean filters. Filters retrieved from our sampler are concentrated into a smaller, surveyable area using high-purity water and dried before they are sent to UH Mānoa's Advanced Election Microscopy Center (AEMC) for analyses.

We used Scanning Electron Microscopy (SEM) and Energy Dispersive X-ray Spectroscopy (EDS) to image and analyze 152 particles from a filter that was exposed before the 2018 Kilauea eruption. The morphologies and elemental spectra of the particles allowed us to assess and categorize the background particle population. We determined that approximately $6 \%$ of the particles are ET particle candidates based on their elemental compositions. Candidates are first identified by composition alone. They include particles that are single mineral grains with compositions inconsistent with local volcanic origin and those having compositions that fall within a factor of 5 of the CI chondrite composition. The wide initial composition range ensures that candidates are not excluded due to uncertainties from occasional physical overlap of grains on the concentrated filter. They are then further classified by their morphology and chemistry. We found a significant fraction of weathered volcanic material, as expected for this collection site. Comparison of the washed particle population with an unwashed and unconcentrated particle population, in progress, is allowing us to assess the potential loss of soluble particle components and particle break-up in our current concentration process. 